\title{
Decision Making Problem Solving using Fuzzy Networks with Rule Base Aggregation
}

\author{
Abdul Malek Yaakob ${ }^{1,2}$ \\ ${ }^{1}$ School of Quantitative Sciences, \\ Universiti Utara Malaysia, \\ 06010 Kedah, Malaysia \\ abd.malek@uum.edu.my
}

\author{
Alexander Gegov ${ }^{2}$ \\ ${ }^{2}$ School of Computing, \\ University of Portsmouth, \\ PO1 3HE United Kingdom \\ alexander.gegov@port.ac.uk
}

\author{
Siti Fatimah Abdul Rahman ${ }^{3}$ \\ ${ }^{3}$ Dept. of Mathematics and Statistics, \\ Universiti Teknologi MARA (Perlis) \\ 02600 Arau, Perlis, Malaysia. \\ sitifatimah471@perlis.uitm.edu.my
}

\begin{abstract}
This paper presents a novel extension of the Technique for Ordering of Preference by Similarity to Ideal Solution (TOPSIS) method. The method is based on aggregation of rules with different linguistic values of the output of fuzzy networks to solve multi criteria decision-making problems whereby both benefit and cost criteria are presented as subsystems. Thus the decision maker evaluates the performance of each alternative for decision process and further observes the performance for both benefit and cost criteria. The aggregation of rule bases in a fuzzy system maps the fuzzy membership functions for all rules to an aggregated fuzzy membership function representing the overall output for the rules. This approach improves significantly the transparency of the TOPSIS methods, while ensuring high effectiveness in comparison to established approaches. To ensure practicality and effectiveness, the proposed method is further tested on equity selection problems. The ranking produced by the method is comparatively validated using Spearman rho rank correlation. The results show that the proposed method outperforms the existing TOPSIS approaches in terms of ranking.
\end{abstract}

Keywords- decision making; fuzzy networks; selection alternatives; fuzzy sets; equity selection, spearman rho.

\section{INTRODUCTION}

Fuzzy systems that incorporate networked rule bases are known as fuzzy networks (FNs). FNs are initiated as a theoretical concept in [1] and are considered to be of white box nature where the inputs are mapped to the outputs through intermediate variables. According to [1], the accuracy of a single rule base is modest but the level of transparency is low, while multiple rule bases are regarded as having low accuracy in dealing among complex decision processes [2]. While in most decision analysis tasks a single rule base and multiple rule bases are common approaches, in this research the emphasis is on FNs as they are highly transparent and moderately accurate.

Therefore FNs consider the collaboration between subsystems. This ability produces considerable benefits to modelling decision processes, and although FNs have been introduced recently, a cohort of researchers are dedicated to the theoretical development and applications of FNs [1], [3]. On the other hand, the reliability of decision information and the experience of experts are still in need of better incorporation into modelling complex decision-making processes [4]. For instance, how self-confident in their choices are stakeholders as decision makers and how much knowledge experts as financial analysts have in appropriate asset classes and markets [5]. Moreover, established TOPSIS methods have a very low transparency level and consequently are not able to trace the performance of benefit and cost measures [6]. In decisionmaking processes, it is vital that decision makers are conscious of how the multiple criteria are performing. The inadequacies above convey the motivation of this study.

The paper is organized as follows: The novel methodology using fuzzy networks with aggregation of rule bases, namely AFN-TOPSIS, is formulated in Section II. Section III illustrates the practicality and effectiveness of proposed method to the problem of traded equity. Further analysis of results and conclusion are provided in Section IV.

\section{NOVEL FUZZY NETWORK METHOD}

A fuzzy network is a new type of fuzzy system, which consists of networked rule bases (nodes) and deals with inputs sequentially, while taking into account the connections and structure of the system [1]. The rules for both fuzzy systems and fuzzy networks are derived from expert knowledge or data. A networked fuzzy system is transparent and fairly accurate at the same time due to its hybrid nature, which facilitates the understanding of decision processes [7]. A rule base is incorporated as a node within the fuzzy network. In this approach, the decision makers' opinions are assessed independently, since they may have varied level of influence degrees, depending on their knowledge in the area.

Additionally, criteria are classified into benefit criteria or cost criteria. Each class will generate correspondingly benefit fuzzy systems or cost fuzzy systems, where the outputs of the systems are Benefit Levels (BL) or Cost Levels (CL), representing the performance of each class. Figure 1 shows the proposed Generalised Fuzzy Network Model for TOPSIS, where Benefit System (BS), Cost System (CS) and Alternative System (AS) are incorporated in the form of fuzzy network nodes. The inputs are the benefit criteria $B_{1} \ldots \mathrm{B}_{\mathrm{e}}$ and the cost criteria $C_{1} \ldots \mathrm{C}_{\mathrm{f}}$. At the end of the process, Alternative Levels (AL) are determined. 


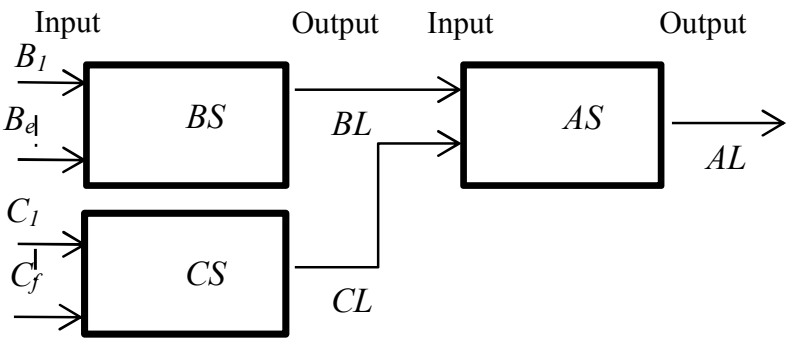

Fig. 1: Fuzzy network model for TOPSIS

The procedures involved in applying a fuzzy network with aggregation rule bases to TOPSIS. Steps 1-6 are adopted from [8] and [9], while steps 7-8 are presented as part of the proposed method in this paper.

Step 1:Construct decision matrices and categorise into two Criteria Categories as Benefit Criteria and Cost Criteria defined through a Benefit System and a Cost System.

In the decision matrices $D_{k}^{B}, D_{k}^{C}$ and weight matrices $W_{k}^{B}, W_{k}^{C}$ $(k=1, \cdots, K)$, it is assumed that $e$ is the number of benefit criteria and $f$ is the number of cost criteria, as shown in Eq. (1):

$$
\begin{aligned}
& D_{k}^{B}=\begin{array}{c}
B_{1} \\
B_{2} \\
\vdots \\
B_{e}
\end{array}\left[\begin{array}{cccc}
x_{11, k} & x_{12, k} & \cdots & x_{1 m, k} \\
x_{21, k} & x_{22, k} & \cdots & x_{2 m, k} \\
\vdots & \vdots & \ddots & \vdots \\
x_{e 1, k} & x_{e 2, k} & \cdots & x_{e m, k}
\end{array}\right] \\
& D_{k}^{C}=\begin{array}{c}
C_{1} \\
C_{2} \\
\vdots \\
C_{f}
\end{array}\left[\begin{array}{cccc}
y_{11, k} & y_{12, k} & \cdots & y_{1 m, k} \\
y_{21, k} & y_{22, k} & \cdots & y_{2 m, k} \\
\vdots & \vdots & \ddots & \vdots \\
y_{f 1, k} & y_{f 2, k} & \cdots & y_{f m, k}
\end{array}\right] \text {; } \\
& W_{k}^{B}=\left[\begin{array}{llll}
g_{1, k} & g_{2, k} & \cdots & g_{e, k}
\end{array}\right] \\
& W_{k}^{C}=\left[\begin{array}{llll}
h_{1, k} & h_{2, k} & \cdots & h_{f, k}
\end{array}\right] \text {, for } k=1, \cdots, K \text {. }
\end{aligned}
$$

and

and

where $x_{i j, k}$ are type-1 fuzzy sets representing the rating of alternatives $A_{j}(j=1, \cdots, m)$ with respect to benefit criteria $B_{i} \quad(i=1, \cdots, e)$ according to the $k^{\text {th }}$ decision maker, and $g_{i, k}$ are type-1 fuzzy sets representing the weights of benefit criteria $B_{i, k}(i=1, \cdots, e)$ according to the $k^{\text {th }}$ decision maker, where $k=1, \cdots, K$. Also, $y_{i j, k}$ are type-1 fuzzy sets describing the rating of alternatives $A_{j}(j=1, \cdots, m)$ with respect to cost criteria $C_{i}(i=1, \cdots, f)$ according to the $k^{\text {th }}$ decision maker, and $h_{i, k}$ are type-1 fuzzy sets describing the weights of cost criteria $C_{i}(i=1, \cdots, f)$ according to the $k^{\text {th }}$ decision maker, where $k=1, \cdots, K$.

Step 2:Construct weighted and normalized decision matrices.
The fuzzy rating and weight of each criterion are variables described with type-1 trapezoidal fuzzy numbers. The ratings of alternatives $A_{j}(j=1, \cdots, m)$ are described with the type-1 trapezoidal fuzzy numbers $x_{i j, k}=\left(a_{i j, k}^{x}, b_{i j, k}^{x}, c_{i j, k}^{x}, d_{i j, k}^{x}\right)$ and $y_{i j, k}=\left(a_{i j, k}^{y}, b_{i j, k}^{y}, c_{i j, k}^{y}, d_{i j, k}^{y}\right)$, while the importance of benefit criteria $B_{i} \quad(i=1, \cdots, e)$ and cost criteria $C_{i}(i=1, \cdots, f)$ are respectively represented by $g_{i, k}=\left(a_{i, k}^{g}, b_{i, k}^{g}, c_{i, k}^{g}, d_{i, k}^{g}\right)$ and $h_{i, k}=\left(a_{i, k}^{h}, b_{i, k}^{h}, c_{i, k}^{h}, d_{i, k}^{h}\right)$, for $k=1, \cdots, K$. The normalized fuzzy decision matrices $R_{k}$ and weight normalized fuzzy decision matrices $V_{k}$ are calculated as shown in Eq. (2):

$R_{k}=\left[r_{i j, k}\right]_{(e+f) \times m}$,

Where

$r_{i j, k}=\left\{\begin{array}{l}r_{i j . k}^{B}=\left(\frac{a_{i j, k}^{x}}{d_{i, k}^{x^{*}}}, \frac{b_{i j, k}^{x}}{d_{i, k}^{x^{*}}}, \frac{c_{i j, k}^{x}}{d_{i, k}^{x^{*}}}, \frac{d_{i j, k}^{x}}{d_{i, k}^{x^{*}}}\right), \text { for } B_{i} \in B \\ r_{i j, k}^{C}=\left(\frac{a_{i, k}^{y^{*}}}{d_{i j, k}^{y}}, \frac{a_{i, k}^{y^{*}}}{c_{i j, k}^{y}}, \frac{a_{i, k}^{y^{*}}}{b_{i j, k}^{y}}, \frac{a_{i, k}^{y^{*}}}{a_{i j, k}^{y}}\right), \text { for } C_{i} \in C\end{array}\right.$

$d_{i, k}^{x^{*}}=\max _{j} d_{i j, k}^{x},(i=1, \cdots, e),(j=1, \cdots, m)$

$a_{i, k}^{y^{*}}=\min _{j} a_{i j, k}^{y}, \quad(i=1, \cdots, f),(j=1, \cdots, m)$

$B$ and $C$ are the sets of benefit criteria and cost criteria respectively;

$V_{k}=\left[v_{i j, k}\right]_{(e+f) \times m}$,

where

$v_{i j, k}= \begin{cases}v_{i j . k}^{B}=r_{i j, k}(\cdot) g_{i, k} & , \text { for } B_{i} \in B \\ v_{i j . k}^{C}=r_{i j, k}(\cdot) h_{i, k} & , \text { for } C_{i} \in C\end{cases}$

and

$v_{i j, k}=\left(a_{i j, k}^{v}, b_{i j, k}^{v}, c_{i j, k}^{v}, d_{i j, k}^{v}\right)$ are type-1 fuzzy sets;

for $k=1, \cdots, K$.

Step 3: Find the Fuzzy Positive Ideal Solution (FPIS) and Fuzzy Negative Ideal Solution (FNIS) for each alternative, and the distance between each alternative to FPIS and FNIS.

The FPIS and FNIS solutions are correspondingly $A_{k}^{+}=\left(v_{1, k}^{+}, v_{2, k}^{+}, \cdots, v_{(e+f), k}^{+}\right)$and $A_{k}^{-}=\left(v_{1, k}^{-}, v_{2, k}^{-}, \cdots, v_{(e+f), k}^{-}\right)$, where $v_{i j, k}^{+}=\left(\begin{array}{llll}1 & 1 & 1 & 1\end{array}\right)$ and $v_{i j}^{-}=\left(\begin{array}{llll}0 & 0 & 0 & 0\end{array}\right)$ are type-1 fuzzy sets, for $k=1, \cdots, K$. The distance for benefit criteria of each alternative $j$ from $A_{k}^{+}$is $\Delta_{j, k}^{B+}$, calculated as shown in Eq. (3):

$$
\Delta_{j, k}^{B+}=\sum_{i=1}^{e} \Delta_{k}^{B}\left(v_{i j, k}^{B}, v_{i, k}^{+}\right), \text {where }
$$




$$
\begin{aligned}
& \Delta_{k}^{B}\left(v_{i j, k}^{B}, v_{i, k}^{+}\right)=\sqrt{\frac{1}{\frac{\left(a_{i j, k}^{v, B}-1\right)^{2}+\left(b_{i j, k}^{v, B}-1\right)^{2}}{3}}+\left(\begin{array}{c}
v, B \\
\left.c_{i j, k}-1\right)^{2}+\left(d_{i j, k}^{v, B}-1\right)^{2}
\end{array}\right]}, \\
& \text { for } j=1, \cdots, m \text {, and } B_{i} \in B \text {, and } k=1, \cdots, K
\end{aligned}
$$

The distance for benefit criteria of each alternative from $A_{k}^{-}$is $\Delta_{j, k}^{B-}$, calculated as shown in Eq. (4):

$$
\begin{aligned}
& \Delta_{j, k}^{B-}=\sum_{i=1}^{e} \Delta_{k}^{B}\left(v_{i j, k}^{B}, v_{i, k}^{-}\right) \text {, where } \\
& \Delta_{k}^{B}\left(v_{i j, k}^{B}, v_{i, k}^{-}\right)=\sqrt{\frac{1}{\frac{1}{3}\left(a_{i j, k}^{v, B}-0\right)^{2}+\left(b_{i j, k}^{v, B}-0\right)^{2}}\left[\begin{array}{l}
\left.c_{i j, k}^{v, B}-0\right)^{2}+\left(d_{i j, k}^{v, B}-0\right)^{2}
\end{array}\right]}, \\
& \text { for } j=1, \cdots, m \text {, and } B_{i} \in B \text {, and } \\
& k=1, \cdots, K \text {. }
\end{aligned}
$$

The distance for cost criteria of each alternative $j$ from $A_{k}^{+}$is $\Delta_{j, k}^{C+}$, calculated as shown in Eq. (5):

$$
\begin{aligned}
& \Delta_{j, k}^{C+}=\sum_{i=1}^{f} \Delta_{k}^{C}\left(v_{i j, k}^{C}, v_{i, k}^{+}\right), \text {where } \\
& \Delta_{k}^{C}\left(v_{i j, k}^{C}, v_{i, k}^{+}\right)=\sqrt{\frac{1}{\frac{1}{3}\left[\begin{array}{l}
\left(a_{i j, k}^{v, C}-1\right)^{2}+\left(b_{i j, k}^{v, C}-1\right)^{2} \\
+\left(c_{i j, k}^{v, C}-1\right)^{2}+\left(d_{i j, k}^{v, C}-1\right)^{2}
\end{array}\right]},}
\end{aligned}
$$$$
\text { for } j=1, \cdots, m \text {, and } C_{i} \in C \text {, and } k=1, \cdots, K
$$

Finally, the distance for cost criteria of each alternative $j$ from $A_{k}^{-}$is $\Delta_{j, k}^{C-}$, calculated as shown in Eq. (6):

$$
\begin{aligned}
& \Delta_{j, k}^{C-}=\sum_{i=1}^{f} \Delta_{k}^{C}\left(v_{i j, k}^{C}, v_{i, k}^{-}\right), \text {where } \\
& \Delta_{k}^{C}\left(v_{i j, k}^{C}, v_{i, k}^{-}\right)=\sqrt{\frac{1}{3}\left[\begin{array}{l}
\left(a_{i j, k}^{v, C}-0\right)^{2}+\left(b_{i j, k}^{v, C}-0\right)^{2} \\
+\left(c_{i j, k}^{v, C}-0\right)^{2}+\left(d_{i j, k}^{v, C}-0\right)^{2}
\end{array}\right]}, \\
& \text { for } j=1, \cdots, m, \text { and } C_{i} \in C \text {, and } k=1, \cdots, K
\end{aligned}
$$

Step 4: Find the closeness coefficients for both the benefit and cost systems.

The closeness coefficients $C C_{j, k}^{B}$ for the benefit systems, and the closeness coefficients $C C_{j, k}^{C}$ for the cost systems, are calculated in Eq. (7):

$$
\begin{gathered}
C C_{j, k}^{B}=\frac{\Delta_{j, k}^{B-}}{\Delta_{j, k}^{B+}+\Delta_{j, k}^{B-}} \\
C C_{j, k}^{C}=\frac{\Delta_{j, k}^{C-}}{\Delta_{j, k}^{C+}+\Delta_{j, k}^{C-}} \\
\text { for } j=1, \cdots, m \text { and } k=1, \cdots, K .
\end{gathered}
$$

Step 5: Derive the Influenced Closeness Coefficients (ICC) by applying the influence degree of each decision maker. Then find the normalised ICC (NICC), dividing the ICC by the maximum value of ICC.

Let $\theta_{k}$ denotes the influence degree, between 0 and 10 (very influential), of decision maker $k$, where $k=1, \cdots, K$. Next, let $\sigma_{k}$ stands for the normalized influence degree of the $k^{\text {th }}$ decision maker, $k=1, \cdots, K$, as evaluated with Eq. (8):

$$
\sigma_{k}=\theta_{k} / \sum_{l=1}^{K} \theta_{l} \quad \text {, for } k=1, \cdots, K .
$$

Eq. (9) evaluates the influence closeness coefficients $I C C_{j, k}^{B}$ and $I C C_{j, k}^{C}$ for each DM $k$, respectively along the benefit and cost criteria.

$$
\begin{aligned}
& I C C_{j, k}^{B}=\sigma_{k} * C C_{j, k}^{B} \\
& \text { and } \quad I C C_{j, k}^{C}=\sigma_{k} * C C_{j, k}^{C} \\
& \text { for } j=1, \cdots, m \text { and } k=1, \cdots, K
\end{aligned}
$$

It is further necessary to normalize the coefficients, to ensure that their values vary between 0 to 1 . Eq. (10) evaluates the normalised coefficients, where $N I C C_{j, k}^{B}$ and $N I C C_{j, k}^{C}$ are respectively the normalized influence closeness coefficients for the benefit and cost systems, as related to the $k^{\text {th }}$ decision maker.

$$
\begin{gathered}
N I C C_{j, k}^{B}=I C C_{j, k}^{B} / \max _{j} I C C_{j, k}^{B} \\
N I C C_{j, k}^{C}=I C C_{j, k}^{C} / \max _{j} I C C_{j, k}^{C} \\
\text { for } j=1, \cdots, m \text { and } k=1, \cdots, K .
\end{gathered}
$$

Both $N I C C_{j, k}^{B}$ and ${ }_{N I C C_{j, k}^{C}}$ will take linguistic terms for the level of alternatives performance.

Step 6: Construct the antecedent matrices and the consequent matrices for the BS and CS systems, based on DMs opinions and the values of the NICC coefficients.

Having the opinions $D_{k}^{B}$ and $D_{k}^{C}$ of all DMs $(k=1, \cdots, K)$ on each alternative $j(j=1, \cdots, m)$ in respect to each benefit criterion $i(i=1, \cdots, e)$ and each cost criterion $i(i=1, \cdots, f)$, we can define the BS antecedent matrix $X_{k}$ and the CS antecedent matrix $Y_{k}$ for each DM $k$, as introduced with Eq. (11):

$$
\begin{gathered}
X_{k}=\left[\begin{array}{cccc}
x_{11, k} & x_{12, k} & \cdots & x_{1 m, k} \\
x_{21, k} & x_{22}, & \cdots & x_{2 m, k} \\
\vdots & \vdots & \ddots & \vdots \\
x_{e 1, k} & x_{e 2, k} & \cdots & x_{e m, k}
\end{array}\right] \text { and } \\
Y_{k}=\left[\begin{array}{cccc}
y_{11, k} & y_{12, k} & \cdots & y_{1 m, k} \\
y_{21, k} & y_{22, k} & \cdots & y_{2 m, k} \\
\vdots & \vdots & \ddots & \vdots \\
y_{f 1, k} & y_{f 2, k} & \cdots & y_{f m, k}
\end{array}\right] \text { for } k=1, \cdots, K
\end{gathered}
$$


where $x_{i j, k}$ and $y_{i j, k}$ are linguistic terms describing decision makers' opinions.

Having determined the $N I C C_{j}^{B, k}$ and $N I C C_{j}^{C, k}$ coefficients for all decision makers $(k=1, \cdots, K)$, next the benefit consequent matrix $\Lambda_{k}$ and the cost consequent matrix $\Psi_{k}$ are defined as shown in Eq. (12):

$$
\begin{aligned}
& \Lambda_{k}=\left[\begin{array}{llll}
\lambda_{1, k} & \lambda_{2, k} & \cdots & \lambda_{m, k}
\end{array}\right] \\
& \Psi_{k}=\left[\begin{array}{llll}
\psi_{1, k} & \psi_{2, k} & \cdots & \psi_{m, k}
\end{array}\right] \\
& \text { for } k=1, \cdots, K \\
& \text { where } \lambda_{i, k} \text { and } \psi_{i, k} \text { are linguistic terms } \\
& \text { representing the output of the BS and CS systems, } \\
& \text { based respectively on the values of } \operatorname{NICC}_{j, k}^{B} \text { and } \\
& \operatorname{NICC}_{j, k}^{C} .
\end{aligned}
$$

The benefit system consists of $K$ matrix decision rules presented in Eq. (13):

$$
\begin{gathered}
\text { If } \quad X_{k}=\left[\begin{array}{cccc}
x_{11, k} & x_{12, k} & \cdots & x_{1 m, k} \\
x_{21, k} & x_{22}, & \cdots & x_{2 m, k} \\
\vdots & \vdots & \ddots & \vdots \\
x_{e 1, k} & x_{e 2, k} & \cdots & x_{e m, k}
\end{array}\right] \\
\Lambda_{k}=\left[\begin{array}{llll}
\lambda_{1, k} & \lambda_{2, k} & \cdots & \lambda_{m, k}
\end{array}\right] \text { for } k=1, \cdots, K ;
\end{gathered}
$$

and can be described with the rule bases in Eq. (14):

Rule 1 : If $B_{1}$ is $x_{11, k}$ and $\cdots$ and $B_{e}$ is $x_{e 1, k}$ then $B L$ is $\lambda_{1, k}$

Rule $m$ : If $B_{1}$ is $x_{1 m, k}$ and $\cdots$ and $B_{e}$ is $x_{e m, k}$ then $B L$ is $\lambda_{m, k}$

where $B L$ is the benefit level of alternatives, for $j=1, \cdots, m$ and , for $k=1, \cdots, K$;

The cost system consists of $K$ matrix decision rules presented in Eq. (15):

$$
\begin{gathered}
\text { If } \quad Y_{k}=\left[\begin{array}{cccc}
y_{11, k} & y_{12, k} & \cdots & y_{1 m, k} \\
y_{21, k} & y_{22, k} & \cdots & y_{2 m, k} \\
\vdots & \vdots & \ddots & \vdots \\
y_{f 1, k} & y_{f 2, k} & \cdots & y_{f m, k}
\end{array}\right] \text { then } \\
\Psi_{k}=\left[\begin{array}{llll}
\psi_{1, k} & \psi_{2, k} & \cdots & \psi_{m, k}
\end{array}\right] \text { for } k=1, \cdots, K ;
\end{gathered}
$$

and can be described with the rule bases in Eq. (16):

Rule 1 : If $C_{1}$ is $y_{11, k}$ and $\cdots$ and $C_{f}$ is $y_{f 1, k}$

then $C L_{1}$ is $\psi_{1, k}$

$\begin{array}{cc}\vdots & \vdots \\ \text { Rule } m & : \text { If } C_{1} \text { is } y_{f 1, k} \text { and } \cdots \text { and } C_{f} \text { is } y_{f m, k}\end{array}$ then $C L_{m}$ is $\psi_{m, k}$

where $C L$ is the cost level of alternatives, for

$$
j=1, \cdots, m \text { and for } k=1, \cdots, K ;
$$

Step 7: Construct the antecedent matrices and consequent matrices for the Alternatives System (AS).

The AS antecedent matrices $\mathrm{M}_{k}$ are based on the Benefit Levels $\Lambda_{k}$ and Cost Levels $\Psi_{k}$, which are the outputs of the BS and CS systems correspondingly. In this case each tuple of inputs $\left(\lambda_{j, k}, \psi_{j, k}\right)$ stands for the assessed levels of the same alternative $j$ through two types of criteria - benefits and costs. Therefore, the AS antecedent matrices $\mathbf{M}_{k}$ are of size $2 \times m$, as constructed in Eq. (18):

$$
\begin{aligned}
M_{k} & =B L\left[\begin{array}{rrrrr}
\lambda_{1, k} & \lambda_{2, k} & \lambda_{3, k} & \cdots & \lambda_{m, k} \\
\psi_{1, k} & \psi_{2, k} & \psi_{3, k} & \cdots & \psi_{m, k}
\end{array}\right] \quad \text { for } \\
k=1, \cdots, K &
\end{aligned}
$$

The AS consequent matrices are derived as follows:

(i)Calculate the aggregation $\xi_{j, k}$ of weighted $N I C C_{j, k}^{B}$ and $N I C C_{j, k}^{C}$, as shown in Eq. (19):

$$
\begin{aligned}
& \xi_{j, k}=\frac{N I C C_{j, k}^{B} \times\left(\frac{e}{e+f}\right)+N I C C_{j, k}^{C} \times\left(\frac{f}{e+f}\right)}{2} \\
& \text { for } j=1, \cdots, m \text { and } k=1, \cdots, K .
\end{aligned}
$$

(ii) Normalize the values of $\xi_{j, k}$ to ensure they lie within $[0,1]$ , as calculated in Eq. (20):

$$
\begin{aligned}
& \quad N \xi_{j, k}=\xi_{j, k} / \max _{j} \xi_{j, k} \quad \text { for } j=1, \cdots, m \text { and } \\
& k=1, \cdots, K .
\end{aligned}
$$

(iii) For $N \xi_{j, k}$, take linguistic terms for the alternatives levels. Then the $K$ AS consequent matrices, in this case of size $1 \times m$ rather than $1 \times m \cdot m$, are described in Eq. (21):

$$
N_{k}=A L\left[\begin{array}{llll}
N \xi_{1, k} & N \xi_{2, k} & \cdots & N \xi_{m, k}
\end{array}\right]
$$

for $k=1, \cdots, K$, where $A L$ is the level of alternatives.

Therefore, the alternatives system is presented with $K$ matrix decision rules, as constructed in Eq. (22):

$$
\begin{aligned}
& \text { If } \quad M_{k}=B L\left[\begin{array}{cccc}
\lambda_{1, k} & \lambda_{1, k} & \cdots & \lambda_{m, k} \\
\psi_{1, k} & \psi_{2, k} & \cdots & \psi_{m, k}
\end{array}\right] \quad \text { then } \\
& N_{k}=A L\left[\begin{array}{llll}
N \xi_{1, k} & N \xi_{2, k} & \cdots & N \xi_{m, k}
\end{array}\right] \text {, } \\
& \text { for } k=1, \cdots, K \text {; }
\end{aligned}
$$

and can be described with the rule bases in Eq. (23):

Rule 1 : If $B L$ is $\lambda_{1, k}$ and $C L$ is $\psi_{1, k}$ then $A L$ is (23) $N \xi_{1, k}$ 
Rule $m$ : If $B L$ is $\lambda_{m, k}$ and $C L$ is $\psi_{m, k}$ then $A L$ is $N \xi_{m, k}$

for $k=1, \cdots, K$; where $B L$ is the level of benefits, $C L$ is the level of costs, and $A L$ is the level of alternatives.

Step 8: Derive the rules for the alternatives based on the generalised decision matrix from Eq. (23), as shown in Eq. (24) for $j=1, \cdots, m$ :

Rule 1: If $B_{1}$ is $x_{1 j, 1}$ and $\cdots$ and $B_{e}$ is $x_{e j, 1}$ and $C_{1}(24)$

is $y_{1 j, 1}$ and $\cdots$ and $C_{f}$ is $y_{f j, 1}$ then $A L$ is $N \xi_{j, 1}$

Rule $n_{j}$ : If $B_{1}$ is $x_{1 j, K}$ and $\cdots$ and $B_{e}$ is $x_{e j, K}$ and

$C_{1}$ is $y_{1 j, K}$ and $\cdots$ and $C_{f}$ is $y_{f j, K}$ then $A L$ is $N \xi_{j, K}$

Step 9: Derive a final score for each alternative.

To produce a final score $\Gamma_{j}$ for each alternative $j$, take the average aggregate membership value of the consequent part of the $n_{j}$ rules in Eq. (24). Then multiply with the influence multiplier based on the $K$ DMs average influence degree for alternative $j$. This is shown in Eq. (25):

$$
\Gamma_{j}=\frac{\sum_{\text {Rule }=1}^{n} N \xi_{j, k}}{n} \bullet \frac{\sum_{k=1}^{K}\left(N I C C_{j, k}^{B}+N I C C_{j, k}^{C}\right)}{K} \quad \text {, for }
$$

Thus, the ranking order of all alternatives can be determined: the better alternatives $j$ have higher values of $\Gamma_{j}$

\section{CASE STUDY: EQUITY MARKET}

In this study, the process of ranking equities follows the proposed methods in Section II with 4 benefit criteria and 2 cost criteria.

Step 1: Based on the information by experts, and using Eq. (1), the decision matrices for the benefit and cost systems can be constructed. The rating of each criterion for each equity and the importance of criteria are based on decision makers' opinions.

Step 2: Considering the benefit system, the normalized decision matrix $R_{k}^{B}$ and the weight normalized decision matrix $V_{k}^{B}$ can be constructed for each $k$, using equations Eq. (2) correspondingly. For example, the calculations for E1 using the opinion of DM1 is as follows:

$$
\begin{aligned}
& r_{11,1}^{B}=(9 / 10,10 / 10,10 / 10,10 / 10)=(0.9,1,1,1) \\
& v_{11,1}^{B}=(0.9 \times 0.9,1 \times 1,1 \times 1,1 \times 1)=(0.81,1,1,1)
\end{aligned}
$$

This step is repeated then for the cost system, to calculate the normalized decision matrix $R_{k}^{C}$ and the weight normalized decision matrix $V_{k}^{C}$.
Step 3: The distances between the rating of criteria for equity and the FPIS and FNIS, can be evaluated as follows. For example, the distance between the first equity E1 per DM1 and the FPIS $A_{1}^{+}$is calculated using Eq. (3) for $j=1$ and $k=1$, as . Next, using Eq. (4) for $j=1$ and $k=1$, the distance between E1 per DM1 and the FPIS $A_{1}^{-}$is calculated as $\Delta_{1,1}^{B-}=1.373+1.063+0.789+1.242=4.4671$. Now, the distances $\Delta_{j, k}^{C+}$ and $\Delta_{j, k}^{C-}$, for example, the distance between the first equity E1 using Eq. (5) as $\Delta_{1,1}^{C+}=\sum_{i=1}^{2} \Delta_{1}^{C}\left(v_{i 1,1}, v_{i, 1}^{+}\right)=0.49+1.12=1.61$

Next, using Eq. (6) for $j=1$ and $k=1$, the distance between E1 according to DM1 and the FPIS $A_{1}^{-}$is calculated as:

$$
\Delta_{1,1}^{C-}=\sum_{i=1}^{2} \Delta_{1}^{C}\left(v_{i 1,1}, v_{i, 1}^{-}\right)=1.017+0.339=1.358
$$

Step 4: Find the closeness coefficients for the benefit system $C C_{j, k}^{B}$ and for the cost system $C C_{j, k}^{C}$, using Eq. (7). For example, the closeness coefficient for E1 in the benefit system under the first decision maker $k=1$ is calculated as follows:

$$
C C_{1,1}^{B}=\frac{\Delta_{1,1}^{B-}}{\Delta_{1,1}^{B+}+\Delta_{1,1}^{B-1}}=\frac{4.4671}{1.4841+4.4671}=0.751
$$

and the closeness coefficient in the cost system

$$
C C_{1,1}^{C}=\frac{\Delta_{1,1}^{C-}}{\Delta_{1,1}^{C+}+\Delta_{1,1}^{C-}}=\frac{1.358}{1.61+1.358}=0.457
$$

Step 5: The Influenced Closeness Coefficients $I C C_{j, k}^{B}$ and $I C C_{j, k}^{C}$ are derived by applying the influence degree $\theta_{k}$. Using Eq. (8) and Eq. (9). Then the normalized coefficients $N I C C_{j, k}^{B}$ and $N I C C_{j, k}^{C}$ are calculated with Eq. (10). For example, the influence degree of DM1 is $\theta_{1}=8$, and using Eq. (8) his normalised expertise. Then the Influenced Closeness Coefficient $I C C_{1,1}^{B}$ for the benefit system for equity E1 according to DM1 is calculated with Eq. (9) and similarly, the corresponding Influenced Closeness Coefficient for the cost system $I C C_{1,1}^{C}$ is produced. Next, the influenced closeness coefficients must be normalized prior to matching the coefficients to the linguistic terms. Using Eq. (10), $N I C C_{1,1}^{B}$ and $N I C C_{1,1}^{C}$ are calculated. Finally, the normalised coefficients are matched to the variable.

Step 6: The antecedent matrices $X_{k}$ for the benefit system are constructed using Eq. (11), based on DM $k$ opinions. The 
consequent matrices $\Lambda_{k}$ for the benefit system are constructed using Eq. (12), based on the values of $N I C C_{j, k}^{B}$ calculated at Step 5 and matched to the linguistic terms. The rule base of the benefit system for DM1 is constructed using Eq. (13) and Eq. (14). By analogy, the rule base for the cost system is constructed.

Step 7: The antecedent matrices $M_{k}$ of each DM $k$ for ES are constructed using Eq. (17) based on the Benefit Level (BL) and Cost Level (CL). Each decision maker has a separate equity antecedent matrix $M_{k}$. Next, the ES consequent matrices $N_{k}$ are derived using Eq. (19)-(21). For example, based on the benefit and cost levels BL and CL evaluated in Step 6 above and using Eq. (18), the ES antecedent matrix $M_{1}$ according to DM1 is evaluated. Next, the ES consequent matrix $N_{1}$ according to DM1 is derived.

Step 8: The final score for each alternative $j=1, \ldots, 25$ is derived with Eq. (25). For example, there are 3 active rules for E1 generated. Eq. (25) is used to obtained final score for E1, the average aggregate membership value for the output of the 3 rules is calculated, and then multiplied with the influence multiplier for E1 across all DMs.

$$
=\frac{0.9+0.9+0.09}{3} \bullet \frac{0.961+0.829+0.932}{3}=0.8162
$$

The ranking positions for all 25 equities considered in this case study is defined based on the higher final score, the better ranking position.

\section{ANALYSIS OF RESULTS AND CONCLUSION}

The established and novel methods are applied to evaluate the score and final ranking of the equities for the case study in section III. Based on the Spearman rho $(\rho)$ analysis [10], it is observed from Table I that the novel method (NM) - namely Aggregation Fuzzy Network (AFN) approach - outperforms the established TOPSIS methods (EM).

This paper introduces a novel TOPSIS method - AFNTOPSIS - that extends the capabilities of rule-based fuzzy networks within multi-criteria decision-making analysis. AFNTOPSIS and incorporates expert knowledge into decision analysis as well as expert degree of experience and influence. At the same time, the approach improves transparency of decision analysis; particularly in the TOPSIS process, by explicitly considering all subsystems and interactions among them. The performance of the proposed method is validated using a benchmark based comparison against two of established methods. The results show that the proposed method AFN-TOPSIS outperforms the established TOPSIS methods in terms of ranking performance.

\section{REFERENCES}

[1] A. Gegov, Fuzzy Networks for Complex System:A Modular Rule Base Approach. Springer-Verlag Berlin Heidelberg, 2011.
A. Gegov, F. Arabikhan, D. Sanders, B. Vatchova, and T. Vasileva, "Fuzzy networks with feedback rule bases for complex systems modelling," Int. J. Knowledge-Based Intell. Eng. Syst., 2017.

[3] A. M. Yaakob, A. Serguieva, and A. Gegov, "FN-TOPSIS: Fuzzy Networks for Ranking Traded Equities," IEEE Trans. Fuzzy Syst., no. 99, pp. 1-19, 2016.

[4] F. J. J. Santos and H. A. Camargo, "Decision support systems in multicriteria groups: An approach based on fuzzy rules," Int. Conf. Fuzzy Syst., pp. 1-8, Jul. 2010.

[5] O. Bello, A. M. Yaakob, A. Gegov, C. Teodoriu, J. Oppelt, and J. Holzmann, "Hybrid Intelligent Decision Support System for Drill Rig Performance Analysis and Selection During Well Construction," in International Petroleum Technology Conference, 2016, pp. 1-17.

[6] R. W. Chen, D. Navin-Chandra, and Fritz B. Prinz, "A Cost-Benefit Analysis Model of Product Design for Recyclability and its Application," IEEE Trans. Compon. Packaging. Manuf. Technol., vol. 17 , no. 4,1994

[7] A. M. Yaakob, A. Gegov, M. Bader-El-Den, and S. F. Abdul Rahman, "Fuzzy systems with multiple rule bases for selection of alternatives using TOPSIS," in 2016 IEEE International Conference on Fuzzy Systems (FUZZ-IEEE), 2016, pp. 2083-2090.

C.-T. Chen, "Extensions of the TOPSIS for group decision-making under fuzzy environment," Fuzzy Sets Syst., vol. 114, no. 1, pp. 1-9, Aug. 2000.

[9] A. M. Yaakob and A. Gegov, "Fuzzy rule based approach with znumbers for selection of alternatives using TOPSIS," in 2015 IEEE International Conference on Fuzzy Systems (FUZZ-IEEE), 2015, pp. $1-8$.

[10] A. M. Yaakob and A. Gegov, "Interactive TOPSIS Based Group Decision Making Methodology Using Z-Numbers," Int. J. Comput. Intell. Syst., vol. 9, no. 2, pp. 311-324, Mar. 2016.

Table I: Spearman rank correlation coefficient based on ranking performance

\begin{tabular}{|c|c|c|c|c|c|c|}
\hline & \multicolumn{2}{|c|}{ Non-Fuzzy (EM) } & \multicolumn{2}{c|}{ Non Rule Based (EM) } & \multicolumn{2}{c|}{ AFN (NM) } \\
\hline Equity & $\partial_{i}$ & $\partial_{i}^{2}$ & $\partial_{i}$ & $\partial_{i}^{2}$ & $\partial_{i}$ & $\partial_{i}^{2}$ \\
\hline E1 & 0 & 0 & -2 & 4 & -2 & 4 \\
\hline E2 & -3 & 9 & 1 & 1 & 1 & 1 \\
\hline E3 & 0 & 0 & 0 & 0 & 0 & 0 \\
\hline E4 & 0 & 0 & 1 & 1 & 1 & 1 \\
\hline E5 & -5 & 25 & -5 & 25 & -5 & 25 \\
\hline E6 & 5 & 25 & 5 & 25 & 4 & 16 \\
\hline E7 & 6 & 36 & 5 & 25 & 2 & 4 \\
\hline E8 & 10 & 100 & 6 & 36 & 6 & 36 \\
\hline E9 & -2 & 4 & -2 & 4 & -2 & 4 \\
\hline E10 & 7 & 49 & 6 & 36 & 6 & 36 \\
\hline E11 & -12 & 144 & -6 & 36 & -6 & 36 \\
\hline E12 & 1 & 1 & -4 & 16 & 0 & 0 \\
\hline E13 & 2 & 4 & 3 & 9 & 4 & 16 \\
\hline E14 & -1 & 1 & 0 & 0 & 1 & 1 \\
\hline E15 & -5 & 25 & -5 & 25 & -3 & 9 \\
\hline E16 & 2 & 4 & 3 & 9 & 3 & 9 \\
\hline E17 & 0 & 0 & -3 & 9 & -5 & 25 \\
\hline E18 & -7 & 49 & -7 & 49 & -7 & 49 \\
\hline E19 & 2 & 4 & 4 & 16 & 4 & 16 \\
\hline E20 & -1 & 1 & 1 & 1 & -1 & 1 \\
\hline E21 & 3 & 9 & 0 & 0 & -2 & 4 \\
\hline E22 & -2 & 4 & -3 & 9 & -2 & 4 \\
\hline E23 & 1 & 1 & 1 & 1 & 1 & 1 \\
\hline E24 & 5 & 25 & 4 & 16 & 4 & 16 \\
\hline E25 & -6 & 36 & -3 & 9 & -2 & 4 \\
\hline & & 556 & & 362 & & 318 \\
\hline & $\rho$ & 0.786 & & 0.861 & & 0.878 \\
\hline Ranking Position & 3 & & 2 & & 1 \\
\hline & & & & & & \\
\hline
\end{tabular}

